\title{
BMJ Open Voluntary home and car smoke-free rules in Japan: a cross-sectional study in 2015
}

\author{
Kensaku Shojima, $^{1,2}$ Takahiro Tabuchi ${ }^{\odot}$
}

To cite: Shojima K, Tabuchi T. Voluntary home and car smoke-free rules in Japan: a cross-sectional study in 2015. BMJ Open 2019;9:e024615. doi:10.1136/ bmjopen-2018-024615

- Prepublication history and additional material for this paper are available online. To view these files, please visit the journal online (http://dx.doi. org/10.1136/bmjopen-2018024615).

Received 5 June 2018 Revised 30 November 2018 Accepted 22 January 2019

Q Check for updates

(c) Author(s) (or their employer(s)) 2019. Re-use permitted under CC BY-NC. No commercial re-use. See rights and permissions. Published by BMJ.

${ }^{1}$ Cancer Control Center, Osaka International Cancer Institute, Osaka, Osaka, Japan

${ }^{2}$ General Internal Medicine, Hashimoto Municipal Hospital, Hashimoto, Wakayama, Japan

Correspondence to

Dr Takahiro Tabuchi;

tabuchitak@gmail.com

\begin{abstract}
Objectives Recently, the Tokyo Metropolitan Assembly passed an ordinance prohibiting smoking in private homes and cars if children are present. However, no previous study has investigated existing, voluntary home and car smoke-free rules in Japan. Therefore, we examined prevalence and determinants of comprehensive home and car smoke-free rules.

Design A cross-sectional study.

Setting Internet survey data with adjustments using inverse probability weighting for 'being a respondent in an internet survey'.

Participants 5600 respondents aged 15-69 years in 2015 were analysed to estimate weighted percentages and prevalence ratios (PRs) with 95\% Cls of having comprehensive home and car smoke-free rules.

Main outcome measures Respondents who answered 'smoking is never allowed' in their home and car were defined as having home and car smoke-free rules. Results Overall, $47.0 \%$ (95\% $\mathrm{Cl}=45.8 \%$ to $48.3 \%)$ of respondents implemented comprehensive home and car smoke-free rules. People who agreed with 'smoking relieves stress' were less likely to have comprehensive smoke-free rules ( $\mathrm{PR}=0.76,0.71$ to 0.82 ), especially among ever-users of electronic nicotine delivery systems ( $P R=0.49,0.30$ to 0.81 ). Higher education was significantly associated with higher PR for comprehensive smoke-free rules ( $P R=1.30,1.19$ to 1.41). Living with children was significantly associated with higher PR for smoke-free rules among current smokers than not living with children (PR=2.91, 1.99 to 4.27).

Conclusions In Japan, about $50 \%$ of respondents had voluntary smoke-free rules in the home and car. Information on current voluntary smoke-free rules will be useful as baseline information on home and car smokefree status before enforcement of the 2018 Tokyo home and car smoke-free legislation.
\end{abstract}

\section{INTRODUCTION}

Secondhand smoke (SHS) is one of the most prevalent and preventable risk factors for many health outcomes in non-smoking adults and children. ${ }^{1}$ Worldwide, approximately $35 \%$ of non-smokers are exposed to SHS and 603000 deaths are attributable to SHS annually. ${ }^{2}$ Smoke-free policies are therefore a key driver behind reducing risk of SHS exposure, particularly among non-smokers. ${ }^{3}$ Previous

\section{Strengths and limitations of this study}

No previous study has investigated home and car smoke-free rules in Japan, although the Tokyo Metropolitan Assembly passed an ordinance to prohibit smoking in private homes and cars if children are present.

- Although an internet-based study has limitations in terms of generalisability, we were able to adjust the data to approximate a nationally representative estimate using inverse probability weighting.

- Our finding that attitudes towards smoking and electronic device use status are related to home and car smoke-free rules provides a novel viewpoint.

- One limitation of our study is that its cross-sectional nature limits our conclusions to associations (not causality) between variables and smoke-free rules.

- Another potential limitation is the use of data that were self-reported without validation testing.

studies have suggested that complete indoor smoking bans decrease the risk of SHS-related diseases such as acute coronary syndrome and childhood asthma. ${ }^{45}$ At least 55 countries, comprising almost 1.5 billion people-20\% of the world's population, have comprehensive smoke-free legislation. ${ }^{3}$ However, this legislation does not determine smoke-free rules for private spaces such as the home and car, which are considered to be two of the greatest areas of exposure to SHS. ${ }^{67}$

Several countries, such as Australia, the UK, South Africa and some jurisdictions in Canada and the USA have enacted laws to ban smoking in cars carrying children. ${ }^{8-10}$ This was possible because the space in a car can legally be considered as semi-public space (this same legal consideration enabled the prohibition of mobile phone use while driving) and previous studies had highlighted the considerable health benefits of smokefree rules, especially for children. ${ }^{11-15}$

A recent review paper reported that the majority of studies showed significant increases in voluntary home smoking restrictions after the enactment of legislative bans 
Table 1 Proportion and PRs of home and car smoke-free rules among total subjects

\begin{tabular}{|c|c|c|c|c|}
\hline & $\mathbf{N}$ & $\mathbf{n}$ & $\%(95 \% \mathrm{Cl})$ & $\mathrm{PR}^{\star}(95 \% \mathrm{Cl})$ \\
\hline Total & 5600 & 2635 & 47.0 (45.8 to 48.3$)$ & \\
\hline \multicolumn{5}{|l|}{ Sex } \\
\hline Female & 2762 & 1327 & 48.1 (46.3 to 49.8 ) & 0.82 (0.76 to 0.89$)$ \\
\hline \multicolumn{5}{|l|}{ Age group } \\
\hline $50-69$ & 2141 & 1060 & 49.5 (47.5 to 51.6$)$ & 1.06 (0.93 to 1.22$)$ \\
\hline \multicolumn{5}{|l|}{ Marital status } \\
\hline Married & 3722 & 1854 & 49.8 (48.3 to 51.4$)$ & 1 (reference) \\
\hline Never married & 1590 & 654 & 41.2 (38.9 to 43.5$)$ & 0.85 (0.75 to 0.96$)$ \\
\hline Technical or junior college & 1135 & 568 & 50.1 (47.3 to 52.8 ) & 1.16 (1.06 to 1.28$)$ \\
\hline University & 1356 & 761 & 56.1 (53.6 to 58.6 ) & $1.30(1.19$ to 1.41$)$ \\
\hline \multicolumn{5}{|l|}{ Home-ownership } \\
\hline No & 1331 & 604 & 45.4 (42.9 to 48.0$)$ & 1 (reference) \\
\hline Yes & 4292 & 2041 & 47.6 (46.1 to 49.0$)$ & 1.00 (0.92 to 1.09$)$ \\
\hline \multicolumn{5}{|l|}{ Self-rated health } \\
\hline Good & 5041 & 2381 & 47.2 (45.9 to 48.5$)$ & 1 (reference) \\
\hline Bad & 582 & 264 & 45.4 (41.6 to 49.3$)$ & 0.94 (0.83 to 1.06$)$ \\
\hline \multicolumn{5}{|l|}{ Children (<12 years) } \\
\hline \multicolumn{5}{|c|}{ 'Difficult to smoke in a place where other people do not smoke' } \\
\hline No & 867 & 402 & 46.4 (43.2 to 49.5$)$ & 1 (reference) \\
\hline Yes & 4755 & 2243 & 47.2 (45.8 to 48.5$)$ & 1.10 (0.99 to 1.22$)$ \\
\hline \multicolumn{5}{|l|}{ 'Smoking relieves stress' } \\
\hline No & 1910 & 1202 & 62.9 (60.9 to 65.0$)$ & 1 (reference) \\
\hline Yes & 3713 & 1443 & 38.9 (37.4 to 40.4$)$ & $0.76(0.71$ to 0.82$)$ \\
\hline \multicolumn{5}{|c|}{ 'In general, what is sold to the public is safe' } \\
\hline No & 4430 & 2298 & 51.9 (50.5 to 53.3) & 1 (reference) \\
\hline Yes & 1192 & 347 & 29.1 (26.7 to 31.6$)$ & $0.77(0.69$ to 0.86$)$ \\
\hline \multicolumn{5}{|l|}{ Smoking status } \\
\hline Never & 3085 & 1739 & 56.4 (54.7 to 58.0$)$ & 1 (reference) \\
\hline Former & 1232 & 719 & 58.4 (55.7 to 61.0$)$ & 0.99 (0.91 to 1.08$)$ \\
\hline Current & 1306 & 187 & 14.3 (12.5 to 16.1$)$ & $0.27(0.24$ to 0.32$)$ \\
\hline \multicolumn{5}{|l|}{ E-cigarettes smoking status } \\
\hline Never & 5195 & 2550 & 49.1 (47.8 to 50.4 ) & 1 (reference) \\
\hline Ever & 428 & 95 & 22.2 (18.5 to 25.9 ) & $0.72(0.59$ to 0.88$)$ \\
\hline
\end{tabular}

${ }^{*}$ Adjusted for all variables. Boldface indicates statistical significance of $P<0.05$.

$\mathrm{N}$, total number; $\mathrm{n}$, number of people who have home and car smoke-free rules; PR, prevalence ratio. 
in public places ${ }^{16}$ Although there is no comprehensive nationwide smoke-free legislation in Japan, even for public spaces, the Tokyo Metropolitan Assembly recently passed an ordinance prohibiting smoking, including electronic nicotine delivery systems, in private homes and cars if children are present. ${ }^{1718}$ While the ordinance will not carry penalties for violators, it will work as an incentive and could influence behaviour, even in private spaces such as the home; Tokyo is one of the largest and the most influential cities in the world. To the best of our knowledge, this is the first ordinance in the world to curtail smoking at home, and we, therefore, need to assess the impact of the ordinance in the future. However, to date, no study has investigated home and car smokefree rules in Japan or the cities of Japan. Therefore, our objective in this study was to examine the prevalence and determinants of such rules. Although this study did not evaluate the Tokyo ordinance, the obtained estimates will be useful as baseline information before enforcement of the 2018 Tokyo home and car smoke-free legislation.

\section{METHODS}

\section{Internet survey}

The survey was conducted between 31 January and 17 February 2015 in Japan and data were collected on the first 9000 respondents (actually 9055 ); that is, 500 people aged 15-19 years and 800 people aged 20-29, 30-39, $40-49,50-59$ and 60-69 years for both sexes. Respondents were selected from a large survey panel recruited and managed by a major nationwide internet research agency, Rakuten Research, and invited to participate in our survey. At the time of our survey the overall survey panel comprised 2278733 people, of whom $53.9 \%$ were male, recruited from the entire Japanese population. Panel members represented all social categories, such as education, housing tenure and marital status, as defined by the Census in Japan. All participants gave informed consent. Details have been given in a previous report. ${ }^{19}$

\section{Measures}

Home and car smoke-free rules

Respondents were asked about smoking rules in their home, using the question: "Please choose the option closest to your current smoking rules in your home'. The response options were 'never allowed', 'always allowed', 'allowed only in some places/times', 'do not have a home (eg, nursing home residents)' and 'no opinion'. We classified respondents who chose 'never allowed' as having home smoke-free rules. Respondents who did not have a home $(n=369)$ or an opinion $(n=765)$ were excluded from the analysis for home smoke-free rules.

Respondents were also asked about smoking rules in their cars, using the question: 'Please choose the option closest to your current smoking rules in your car'. The response options were 'never allowed', 'always allowed', 'allowed sometimes', 'do not have a car' and 'no opinion'. We classified respondents who chose 'never allowed' as having a car smoke-free rules. Respondents who did not have a car $(n=1647)$ or an opinion $(n=599)$ were excluded from the analysis for car smoke-free rules.

We classified respondents who chose 'never allowed' for both home and car rules as having comprehensive home and car smoke-free rules. Respondents who had neither a home nor a car $(n=1684)$ or who did not have opinions for home or car rules $(n=956)$ were excluded from the analysis for home and car smoke-free rules. Furthermore, we excluded respondents showing discrepancies and/or artificial/unnatural responses in the analyses. For example, if a respondent chose the same answer number throughout a set of questions, it was deemed to indicate a discrepancy. Further details of discrepancy identification are also shown in previous reports. ${ }^{19} 20$ After excluding respondents with a discrepancy, we analysed 7106 subjects (weighted number $=7165$ ), for smoke-free home rules, 5994 subjects (weighted number=6652) for smoke-free car rules and 5600 subjects (weighted number $=6240$ ) for both rules.

\section{Characteristics of respondents}

Characteristics included: sex, age, marital status (married, never married, widowed and divorced), education (less than high-school and high school, technical or junior college, university [4 years] and graduate college), housing tenure (home-owner or not), self-rated health, living with children (under 12 years) (yes or no), workplace smoke-free rules (yes or no), home smoke-free rules (yes or no), car smoke-free rules (yes or no), smoking status and e-cigarette smoking status and attitudes towards smoking in terms of the following three issues (yes or no): (1) agree with 'it is difficult to smoke in a space where other people do not smoke', (2) agree with 'smoking relieves stress' and (3) agree with 'in general, what is sold to the public is safe'.

\section{Smoking status}

Respondents were asked about combustible cigarette smoking status, using the question: 'Please choose your current status for tailor-made and roll-your-own cigarettes separately'. The response options were 'never user', 'former non-regular user', 'former regular user' and 'current user'. Respondents who currently smoked combustible cigarettes (tailor-made and roll-your-own cigarettes) were considered 'current smokers'. Those who reported former use and did not currently smoke either type of cigarette were considered 'former smokers'. Those who had never smoked were considered 'never smokers'.

\section{Electronic devices use status}

Respondents were asked about their use of each of the following products: nicotine e-cigarettes, non-nicotine e-cigarettes, e-cigarettes with unknown nicotine content, Ploom and IQOS, using the question: 'Please choose your current status for each product', and the response options were 'never user', 'former non-regular user', 'former regular user' and 'current user'. The latter three 


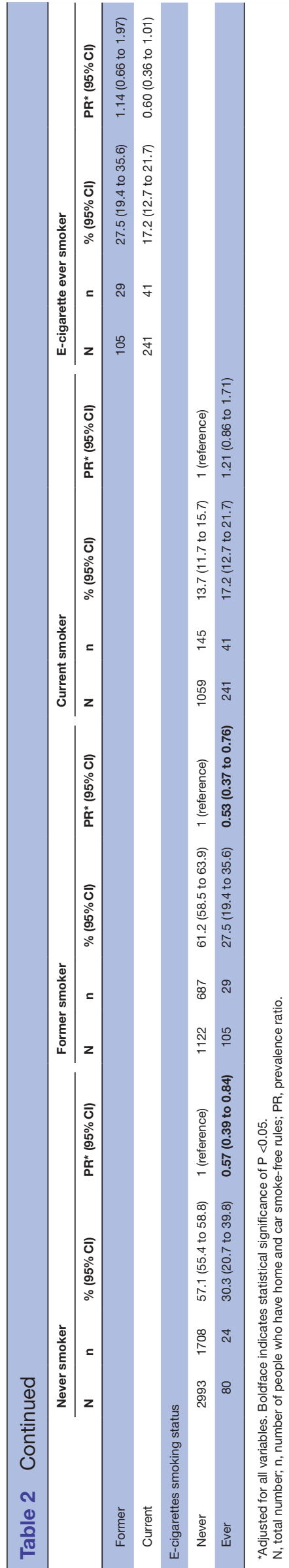

responses were combined and defined as 'ever user' of electronic devices. Those who had never smoked any of the above five products were considered as 'never user' of electronic devices.

\section{Statistical analyses}

Internet surveys are not necessarily representative of the real world because they are not based on completely random sampling. However, previous studies have suggested that estimates adjusted using inverse probability weighting (IPW) obtained from a propensity score (calculated by logistic regression models using basic demographic and socioeconomic factors such as education and housing tenure) from an internet-based convenience sample provide similar estimates of parameters, or at least reduced the differences compared with probability sample based estimates. ${ }^{1921} 22$ We therefore present IPW-adjusted estimates rather than simple internet survey estimates as the main results of this study. To correct for the selectivity of internet-based samples, we used a nationally representative probability sample from the Japanese Comprehensive Survey of Living Conditions of People on Health and Welfare (CSLCPHW) ${ }^{23}$ Data from two surveys (internet survey and CSLCPHW) were pooled (combined) and used for a logistic regression model with all the covariates mentioned above to estimate the probability of 'being a respondent in an internet survey', that is, propensity score. Detailed methods are available from our previous report. ${ }^{19}$

We present weighted percentages and prevalence ratios (PRs) for having home and car smoke-free rules (main outcome), home smoke-free rules and car smokefree rules (online supplementary outcomes) among all respondents according to characteristics such as sex, age group and smoking status. Because the outcomes were not rare, we used log-binomial regression models to calculate the PRs for smoke-free rules, but the models did not converge. Therefore, we used log-Poisson models, which provide consistent but not fully efficient estimates of the PRs (ie, the CIs are slightly wider) ${ }^{24}{ }^{25}$ Percentages are shown with $95 \%$ CIs calculated by Wald and exact methods.

Probability values for statistical tests were two-tailed; $\mathrm{p}<0.05$ was considered statistically significant. All analyses were performed using SAS V.9.3 (SAS Institute).

Patients and public involvement

Patients and public were not involved in the study.

\section{RESULTS}

Overall, $47.0 \%$ (95\% CI $=45.8 \%$ to $48.3 \%)$ of respondents implemented comprehensive home and car smokefree rules (table 1). The prevalence of smoke-free rules by smoking status was highest among former smokers $(58.4 \%)$, followed by never smokers $(56.4 \%)$, then current smokers $(14.3 \%)$. We found that $49.1 \%$ of e-cigarette ever users had smoke-free rules. Whereas, $55.1 \%$ 


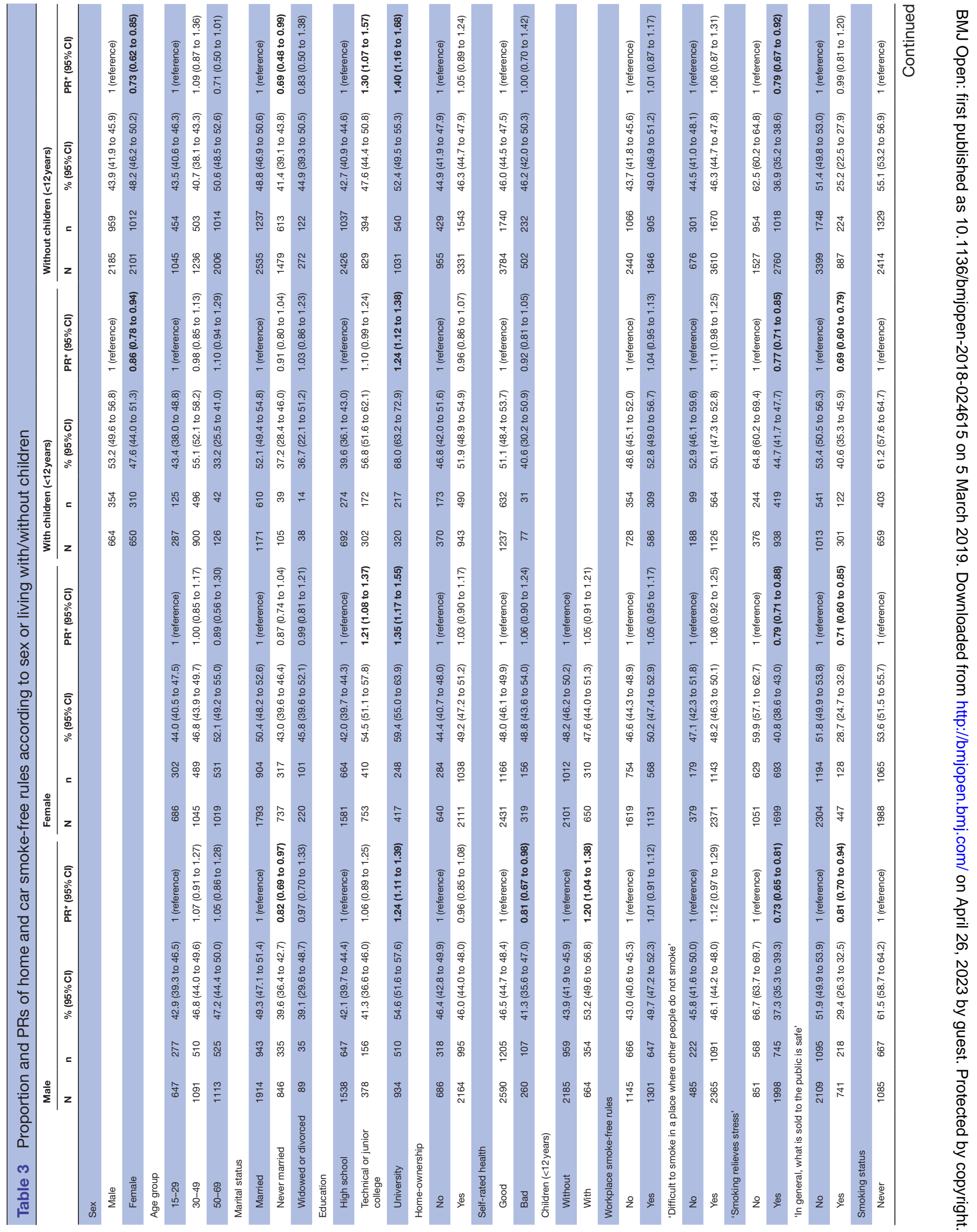




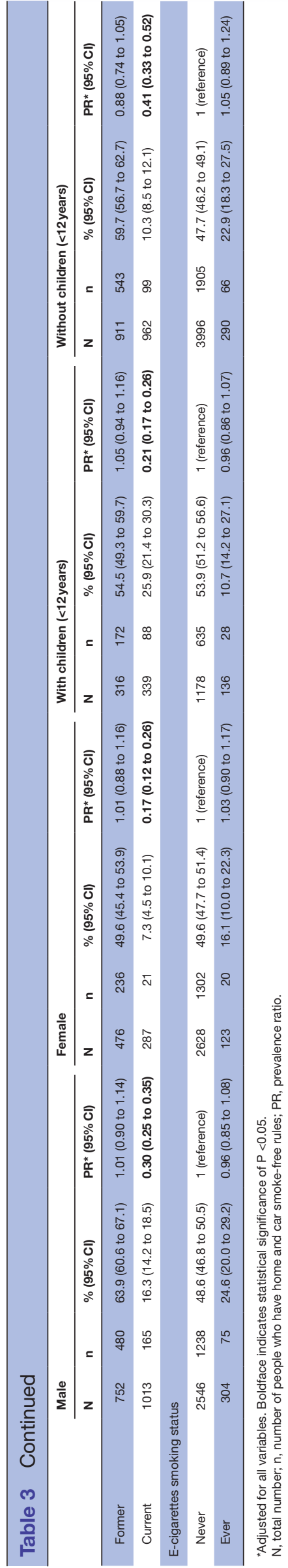

(95\% CI $=53.9 \%$ to $56.2 \%)$ of respondents had home smoke-free rules, and $62.4 \%(95 \% \mathrm{CI}=61.2 \%$ to $63.5 \%)$ of respondents had car smoke-free rules (online supplementary table $\mathrm{S} 1$ ).

Table 1 shows PRs of home and car smoke-free rules among total subjects. Respondents who were 'female', 'never married', agreed with 'smoking relieves stress', agreed with 'in general, what is sold to the public is safe', 'current smokers' and 'electronic device ever user' had significantly lower PRs of comprehensive home and car smoke-free rules than the reference category. Respondents who had 'higher education' and were living with children had significantly higher PRs of comprehensive home and car smoke-free rules than the reference category. Online supplementary table S1 also shows PRs of home or car smoke-free rules among total subjects.

Results stratified by tobacco use status (never, former and current smoker, and e-cigarette ever user) are shown in table 2 and by sex or living with children (male, female and living with or without children) in table 3. Among all tobacco users, people who agreed with 'smoking relieves stress' had significantly lower PRs for comprehensive home and car smoke-free rules. Higher education attainments were significantly associated with higher PRs for smoke-free rules except for former smokers and electronic device ever-users. Differences across tobacco user status were observed in some characteristics. Living with children was significantly associated with a higher PR for smoke-free rules than not living with children, among current smokers. Ever-users of electronic devices had significantly lower PRs than non-users, for home and car smoke-free rules among never and former smokers. Online supplementary tables S2 and S3 for home smokefree rules also show results stratified by tobacco use status and sex or living with children, respectively. Online supplementary tables S4 and S5 for car smoke-free rules show results stratified by tobacco use status and sex or living with children, respectively.

\section{DISCUSSION}

To the best of our knowledge, this is the first study to examine the prevalence and predictors of voluntary home and car smoke-free rules in Japan. Even among non-smokers, coverage of voluntary smoke-free rules $(56.4 \%)$ is far from complete and much lower than that in the USA $(85 \%) .{ }^{26}$ The estimated proportion of households with home and car smoke free rules in Japan $(55.1 \%$ or $62.4 \%)$ was lower than that observed in a previous study in the USA $(83.7 \%$ or $78.1 \%),{ }^{12}$ but higher than that in Spain $(45.6 \%$ or $61.6 \%) .{ }^{27}$ The estimated proportion of current smokers who implemented smoke-free rules in their homes in Japan $(23.5 \%)$ was lower than in Australia (34.1\%), Canada (27.3\%) and the USA (26.4\%), but higher than in the UK $(15.3 \%){ }^{28}$ In households with smokers, children in particular suffer from SHS inhalation in homes and cars. ${ }^{29}$ In the present study, living with children is significantly associated with 
comprehensive home and car smoke-free rules among current smokers. This may suggest that smokers are aware of the harm tobacco caused by tobacco and restrict smoking to protect their children. ${ }^{30}$ Previous studies have reported that smokers who know about the harmful effects of SHS were more likely to have smoke-free home or car rules. ${ }^{132831}$ Public health campaigns are therefore necessary to heighten the public perception of the harmfulness of exposure to SHS in private spaces and, as a result, increase voluntary adoption of smoke-free home and car rules. ${ }^{32}$

Recently, novel electronic nicotine delivery systems such as heat-not-burn tobacco and e-cigarettes have been introduced to the Japanese market. ${ }^{20}$ Use of these products is considered in the present study under the heading 'electronic devices use status'. Our findings suggest that use of electronic devices use might impede the introduction of smoke-free rules at home and in the car (this is the first report to suggest these associations). A previous study that examined the determinants of home and car smoke-free rules among e-cigarette and combustible cigarette users reported the home smoke-free rate among e-cigarette users $(82.5 \%)$. However, this study did not report the association between e-cigarette use and implementation of smoke-free rules for the home and the car. ${ }^{12}$

The finding that women are less likely to have smokefree rules concurs with the previous study. ${ }^{12}$ Although pregnant women are not covered by the Tokyo home and car smoke-free legislation, protecting them is desirable.

Several attitudes towards smoking were also associated with home and car smoke-free rules. Previous studies have revealed that smokers who did not believe cigarette smoke was dangerous and could cause lung cancer in non-smokers were more likely to smoke at home and in the car with non-smokers. ${ }^{132831}$ Further, many people believe 'smoking relieves stress'. This is, however, a false belief, ${ }^{33}$ created by the tobacco industry who funded psychological researchers such as Hans Selye. ${ }^{34}$ If nicotine-dependent people are deprived of the drug, they are stressed until the moment they smoke a cigarette and satisfy the craving, hence the feeling of relief. We have to educate the public about stress and smoking public. This may lead to better understanding about tobacco products, as well as the implementation of smoke-free rules in homes.

There are several limitations to this study. First, due to the cross-sectional nature of the study, conclusions can only be made about associations (not causality) between variables and smoke-free rules. Second, data were self-reported without validation testing. In some cases, smokefree rules in homes and cars might be ambiguous, especially if there are no smokers in the family. Our analysis does not include variables on whether smokers are living together, although this variable has often been used in previous studies. Third, the measures for smoking status were not ideal. We did not collect information on whether respondents had smoked more than 100 cigarettes in their lifetime as a cut-off for regular smoker.
CONCLUSION

About $50 \%$ of the Japanese population had home and car smoke-free rules, although these private spaces have not been included in the smoke-free legislation. This information will be useful as a baseline on home and car smoke-free status before the enforcement of the 2018 Tokyo home and car smoke-free legislation.

Acknowledgements The authors thank Dr Julia Mortimer for her English language editing.

Contributors TT had full access to all of the study data and takes responsibility for the integrity of the data and the accuracy of the data analysis. Study concept and design: KS, TT. Acquisition of data: TT. Analysis and interpretation of data: all authors. Drafting of the manuscript: KS. Critical revision of the manuscript for important intellectual content: KS, TT. Statistical analysis: KS, TT. Study supervision: TT.

Funding This work was supported by Health Labour Sciences Research Grants (H26-junkankitou-ippan-023 and H28-junkankitou-ippan-002), Japan Society for the Promotion of Science (JSPS) KAKENHI Grants (15K19256; 18H03062), and the Japan Agency for Medical Research and Development (Grant number; 27310201).

Disclaimer The funding sources had no role in the design and conduct of the study; in the analysis and interpretation of the data; or in the preparation, review, or approval of the manuscript.

Competing interests None declared.

Patient consent for publication Obtained.

Ethics approval The study was reviewed and approved by the Research Ethics Committee of the Osaka International Cancer Institute (no. 1412175183).

Provenance and peer review Not commissioned; externally peer reviewed.

Data sharing statement Extra data can be accessed via the Dryad data repository at http://datadryad.org/withthedoi:10.5061/dryad.3m5m6hp.

Open access This is an open access article distributed in accordance with the Creative Commons Attribution Non Commercial (CC BY-NC 4.0) license, which permits others to distribute, remix, adapt, build upon this work non-commercially, and license their derivative works on different terms, provided the original work is properly cited, appropriate credit is given, any changes made indicated, and the use is non-commercial. See: http://creativecommons.org/licenses/by-nc/4.0/.

\section{REFERENCES}

1. US Department of Health and Human Services. The health consequences of involuntary exposure to tobacco smoke: a report of the surgeon general. Atlanta, GA: Centers for Disease Control and Prevention, 2006.

2. Oberg M, Jaakkola MS, Woodward A, et al. Worldwide burden of disease from exposure to second-hand smoke: a retrospective analysis of data from 192 countries. Lancet 2011;377:139-46.

3. World Health Organization. WHO Report on the global tobacco epidemic, 2017: monitoring tobacco use and prevention policies. Geneva: WHO, 2017.

4. Pell JP, Haw S, Cobbe S, et al. Smoke-free legislation and hospitalizations for acute coronary syndrome. N Engl J Med 2008;359:482-91.

5. Mackay D, Haw S, Ayres JG, et al. Smoke-free legislation and hospitalizations for childhood asthma. N Engl J Med 2010;363:1139-45.

6. Centers for Disease Control and Prevention (CDC). Vital signs: nonsmokers' exposure to secondhand smoke - United States, 19992008. MMWR Morb Mortal Wkly Rep 2010;59:1141-6.

7. Tabuchi T, Nakamura M. Disparity of secondhand smoke exposure at home and/or workplace according to age, education and medical insurance in Japan. JACR Monogr 2014;20:39-48.

8. Kruger J, Jama A, Kegler M, et al. Smoke-free rules and secondhand smoke exposure in vehicles among U.S. Adults - National Adult Tobacco Survey, 2009-2010 and 2013-2014. Int J Environ Res Public Health 2016;13:1048.

9. Wise J. Smoking in cars carrying children will be illegal in England from October. BMJ 2015;350:h836.

10. Canadian Cancer Society. Laws banning smoking in vehicles carrying children-international overview. 2011. 
11. Mills AL, Messer K, Gilpin EA, et al. The effect of smoke-free homes on adult smoking behavior: a review. Nicotine Tob Res 2009;11:1131-41.

12. Kruger J, Jama A, Homa DM, et al. Smoke-free home and vehicle rules by tobacco use status among US adults. Prev Med 2015;78:9-13.

13. Hitchman SC, Fong GT, Borland R, et al. Predictors of smoking in cars with nonsmokers: findings from the 2007 Wave of the International Tobacco Control Four Country Survey. Nicotine Tob Res 2010;12:374-80.

14. Hitchman SC, Guignard R, Nagelhout GE, et al. Predictors of car smoking rules among smokers in France, Germany and the Netherlands. Eur J Public Health 2012;22:17-22.

15. Hopkinson NS, Majeed A, Britton J, et al. Respiratory health professionals call on MPs to vote to ban smoking in cars with children. BMJ 2014;348:g1395.

16. Monson E, Arsenault N. Effects of Enactment of Legislative (Public) Smoking bans on voluntary home smoking restrictions: a review. Nicotine Tob Res 2017;19:141-8.

17. Nomura S. Tokyo moves to protect kids from passive smoking at home, in cars. in Japanese: The Asahi Shimbun, 2017.

18. The Tokyo Metropolitan Government. The ordinance aimed at protecting children from inhaling secondhand smoking in homes and other places. Tokyo: The Tokyo Metropolitan Government, 2017.

19. Tabuchi T, Kiyohara K, Hoshino T, et al. Awareness and use of electronic cigarettes and heat-not-burn tobacco products in Japan. Addiction 2016;111:706-13.

20. Tabuchi T, Gallus S, Shinozaki T, et al. Heat-not-burn tobacco product use in Japan: its prevalence, predictors and perceived symptoms from exposure to secondhand heat-not-burn tobacco aerosol. Tob Control 2018;27:e25-33.

21. Hoshino T. Statistics for observational data and surve data-Causal inference, selection bias and data fusion. Tokyo: Iwanami Shoten, 2009.

22. Schonlau M, van Soest A, Kapteyn A, et al. Selection bias in web surveys and the use of propensity scores. Sociol Methods Res 2009;37:291-318
23. Ministry of Health, Labour and Welfare. Comprehensive survey of living condition of people on health and welfare. Tokyo, 2014

24. McNutt LA, Wu C, Xue X, et al. Estimating the relative risk in cohort studies and clinical trials of common outcomes. Am J Epidemiol 2003;157:940-3.

25. Zou G. A modified poisson regression approach to prospective studies with binary data. Am J Epidemiol 2004;159:702-6.

26. Parks MJ, Kingsbury JH, Boyle RG, et al. Smoke-free rules in homes and cars among smokers and nonsmokers in minnesota. Prev Chronic Dis 2018:15:17035.

27. Díez-Izquierdo A, Lidón-Moyano C, Martín-Sánchez JC, et al. Smoke-free homes and attitudes towards banning smoking in vehicles carrying children in Spain (2016). Environ Res 2017;158:590-7.

28. Borland $\mathrm{R}$, Yong $\mathrm{HH}$, Cummings $\mathrm{KM}$, et al. Determinants and consequences of smoke-free homes: findings from the International Tobacco Control (ITC) Four Country Survey. Tob Control 2006;15:iii42-50.

29. International Agency for Research on Cancer. IARC handbooks of cancer prevention, tobacco control, volume 13: evaluating the effectiveness of smoke-free policies. Lyon, France: International Agency for Research on Cancer, 2009.

30. Rosen LJ, Noach MB, Winickoff JP, et al. Parental smoking cessation to protect young children: a systematic review and meta-analysis. Pediatrics 2012;129:141-52.

31. Hitchman SC, Fong GT, Zanna MP, et al. Support and correlates of support for banning smoking in cars with children: findings from the ITC Four Country Survey. Eur J Public Health 2011;21:360-5.

32. Lidón-Moyano C, Martínez-Sánchez JM, Fu M, et al. Secondhand smoke risk perception and smoke-free rules in homes: a crosssectional study in Barcelona (Spain). BMJ Open 2017;7:e014207.

33. Halterman JS, Fagnano M, Conn KM, et al. Barriers to reducing ETS in the homes of inner-city children with asthma. J Asthma 2007;44:83-8.

34. Petticrew MP, Lee K. The "father of stress" meets "big tobacco": Hans Selye and the tobacco industry. Am J Public Health 2011:101:411-8. 